\title{
Reduced intrinsic connectivity of amygdala in adults with major depressive disorder
}

\section{Rajamannar Ramasubbu ${ }^{1,2,3,4}$ *, Nithya Konduru ${ }^{1}$, Filomeno Cortese ${ }^{4}$, Signe Bray ${ }^{5,6}$, Ismael Gaxiola-Valdez ${ }^{4}$ and Bradley Goodyear ${ }^{1,2,4,5}$}

' Department of Psychiatry, University of Calgary, Calgary, AB, Canada

${ }^{2}$ Department of Clinical Neuroscience, University of Calgary, Calgary, AB, Canada

${ }^{3}$ Mathison Centre for Mental Health Research and Education, University of Calgary, Calgary, AB, Canada

${ }^{4}$ Hotchkiss Brain Institute, University of Calgary, Calgary, AB, Canada

${ }^{5}$ Department of Radiology, University of Calgary, Calgary, $A B$, Canada

${ }^{6}$ Alberta Children's Hospital Research Institute, Calgary, AB, Canada

\section{Edited by:}

Andreas Jansen, Philipps-University Marburg, Germany

Reviewed by:

Martin Walter,

Otto-von-Guericke-Universität

Magdeburg, Germany

Stefan Frässle, Philipps-University

Marburg, Germany

*Correspondence:

Rajamannar Ramasubbu, Department of Psychiatry/Clinical Neurosciences, Mathison Centre for Mental Health Research and Education, University of Calgary, TRW building, Room 4D64, 3280 Hospital Drive NW, Calgary, AB T2N4Z6, Canada

e-mail: rramasub@ucalgary.ca
Imaging studies of major depressive disorder (MDD) have demonstrated enhanced restingstate activity of the amygdala as well as exaggerated reactivity to negative emotional stimuli relative to healthy controls (HCs). However, the abnormalities in the intrinsic connectivity of the amygdala in MDD still remain unclear. As the resting-state activity and functional connectivity (RSFC) reflect fundamental brain processes, we compared the RSFC of the amygdala between unmedicated MDD patients and HCs. Seventy-four subjects, 55 adults meeting the DSM-IV criteria for MDD and $19 \mathrm{HCs}$, underwent a resting-state 3-T functional magnetic resonance imaging scan. An amygdala seed-based low frequency RSFC map for the whole brain was generated for each group. Compared with HCs, MDD patients showed a wide-spread reduction in the intrinsic connectivity of the amygdala with a variety of brain regions involved in emotional processing and regulation, including the ventrolateral prefrontal cortex, insula, caudate, middle and superior temporal regions, occipital cortex, and cerebellum, as well as increased connectivity with the bilateral temporal poles $(p<0.05$ corrected). The increase in the intrinsic connectivity of amygdala with the temporal poles was inversely correlated with symptom severity and anxiety scores. Although the directionality of connections between regions cannot be inferred from temporal correlations, the reduced intrinsic connectivity of the amygdala predominantly with regions involved in emotional processing may reflect impaired bottom-up signaling for top-down cortical modulation of limbic regions leading to abnormal affect regulation in MDD.

Keywords: depression, amygdala, fMRI, functional connectivity, neural networks

\section{INTRODUCTION}

Major depressive disorder (MDD) is potentially a life-threatening mood disorder with substantial morbidity and mortality (1-3). It is the leading source of disability and disease burden worldwide (4). Despite advances in pharmacological treatment over the last two decades, the burden of depression continues to rise, as 30-40\% of patients do not respond to antidepressants (5). As MDD is a complex heterogeneous brain disorder with variations in etiology, symptoms, course, and treatment responsiveness, more research is needed to understand the complexity of the underlying neurobiology of this disorder and to identify potential brain targets for treatment optimization.

A large body of evidence from neuroimaging studies suggests that depression is the result of disruptions of neural circuits encompassing large parts of prefrontal cortex (PFC), limbic, and subcortical structures $(6,7)$. The current neural models of depression propose emotional dysregulation due to abnormalities in the functioning of the dorsal neural system, also called the cognitive control system, and ventral neural system, also called the emotion appraisal system (8-10). The dorsal system, which comprises the dorsolateral (dl) PFC, dorsomedial (dm) PFC, dorsal (d) anterior cingulate cortex (ACC), and hippocampus, is involved in cognitive processing of emotional input and effortful or voluntary regulation of emotions. The ventral system, which comprises the amygdala, insula, ventral striatum, ventral ACC, and ventral PFC, is crucial for the identification of emotional significance of internal or external stimuli, generation and automatic regulation (regulation without any conscious effort) of affective states, and mediation of autonomic responses depending on stimuli and context resulting in the production of affective states. It has been proposed that increased activity in the ventral neural system and decreased activity in the dorsal neural system may result in a negative bias with predominance in the attention, identification of negative emotions, and other cognitive and vegetative symptoms of depressive disorder (11).

The amygdala is a critical brain region for both bottom-up and top-down processes of emotion generation and regulation (12). It plays a crucial role in bottom-up processes such as emotional perception and encoding affective, motivational and social salience of environmental stimuli, generation of affective state and 
autonomic responses, directing attention and orienting responses to emotional stimuli, and formation of emotional memory and fear conditioning (13). Studies have identified an increase in amygdala activity in the bottom-up triggering of arousal to potentially threatening stimuli, especially for fearful and angry facial stimuli, even if the observer had no conscious awareness of seeing them $(14,15)$. Furthermore, the amygdala plays a role in emotional regulation through direct modulation of the medial PFC and ACC, which are considered to be important for top-down regulation of emotion (16-18). Studies in MDD also find abnormally increased hemodynamic and metabolic activity of the amygdala in the resting-state $(19,20)$ and in response to negative emotional faces presented explicitly, implicitly, or subliminally compared with healthy controls (HCs) corroborating the negative bias hypothesis of depression $(21,22)$.

As the amygdala interacts extensively within widely distributed cortical-subcortical-limbic circuits, there is increase in number of studies over the last decade examining the functional interactions between the amygdala and other brain regions to understand the disturbances in the functional connections or functional connectivity in amygdala based neural systems in depression. "Functional connectivity" refers to the temporal correlation between the neural activity patterns of anatomically separated brain regions, which is thought to reflect the level of functional communication between the regions (23). Most task-based functional connectivity studies in MDD patients have shown decreased connectivity of the amygdala compared to HCs during negative emotion processing with both dorsal and ventral neural system regions (24-31). However, other studies have shown increased functional connectivity between the amygdala and subgenual cingulate cortex during an emotional face matching task in $\operatorname{MDD}(26,32)$. Although taskrelated functional connectivity studies provide information on how amygdala connectivity is altered during emotional and cognitive processing, it is crucial to know the abnormalities in the functional connectivity of the amygdala during resting conditions in the absence of any explicit external stimulus or tasks (intrinsic connectivity) to fully understand the dysregulation of amygdala based networks and how this may relate to abnormal response patterns and symptoms in patients with MDD.

In recent years, the resting-state functional magnetic resonance imaging (fMRI) approach has been widely used in psychiatry to probe differences in neural circuits between groups without the potential confounds of task performance and practice/habituation effects. Resting-state data can be processed either by: (1) regionof-interest (ROI) or seed-based analysis, or (2) independent components analysis (ICA) (33). The ROI method is a model-based, hypothesis driven approach, wherein a seed region or ROI is selected a priori, and the resulting functional connectivity map is generated from the temporal correlations between the seed region and other brain regions $(23,34)$. ICA is a model-free or data-driven approach wherein the data of the entire brain is decomposed into spatially distinct clusters (i.e., components) exhibiting similar temporal behavior. This approach is particularly useful for studies with no specific hypothesis as to the brain regions involved (35).

The results of resting-state fMRI studies regarding amygdala connectivity in MDD have been inconsistent. Among six restingstate fMRI studies using model-free ICA, only one study showed deceased bilateral amygdala and left insula resting-state activity and functional connectivity (RSFC) in an affective network (36), whereas the other five studies found no abnormalities in amygdala connectivity $(35,37-40)$. Among four resting-state fMRI studies using the ROI approach and the amygdala as a seed, one study showed reduced RSFC between ACC and amygdala in nonrefractory MDD (41), and another study showed decreased negative intrinsic connectivity between the amygdala seed region and fronto-insular region in MDD (42). However, other two studies did not show altered RSFC of the amygdala either in unipolar depression or major depression compared to HCs $(43,44)$. Recently, using NAcc as the ROI, Alexopoulos et al. (45) showed reduced RSFC between NAcc and the amygdala in late-life depression. The discrepancy in findings relating to RSFC of amygdala in MDD may be due to small sample sizes, limiting the RSFC analysis of the amygdala only to few predefined target areas, and heterogeneity in clinical characteristics of the study sample. Given the discrepancies in findings and limitations in the previous literature, more studies are needed to investigate the abnormalities of intrinsic connectivity of the amygdala in MDD in a relatively large sample using seed-to-whole brain connectivity analysis to move forward in this field of research.

The main objective of the current study is to examine the abnormalities in the intrinsic functional connections of the amygdala in a large sample of MDD subjects using a seed-based, whole brain functional connectivity analysis of resting-state fMRI data. The seed-based approach is commonly recommended for studies involving a specific hypothesis with a priori defined region such as amygdala, because of the simplicity in analysis and interpretation of the results (33). Given that the amygdala has intrinsic connectivity with multiple brain regions (46) and intrinsic connectivity between multiple regions is affected in MDD (33), we preferred to examine the abnormalities of RSFC of amygdala using seedto-whole brain connectivity analysis. On the basis of the reviewed literature on amygdala and emotional circuitry disturbances in MDD, we predicted that compared to healthy subjects, patients with MDD would show aberrant intrinsic connectivity of amygdala with prefrontal, limbic, and subcortical regions involved in emotional processing and regulation. Based on the findings from resting-state-fMRI studies of amygdala in MDD, we also predicted that MDD patients would show reduced intrinsic connectivity of amygdala with insula, striatum, and ACC compared to healthy subjects.

\section{MATERIALS AND METHODS SUBJECTS}

Fifty-five patients fluent in English and meeting DSM-IV criteria for MDD (47), according to the Structured Clinical Interview for DSM-IV Axis 1 Disorders (48), were recruited through advertisements. The severity of depressive and anxiety symptoms were assessed using the clinician-administered, 17-item Hamilton rating scale for depression (HRSD) and Hamilton anxiety rating scale (HAM-A), respectively $(49,50)$. Patients were of both genders, right-handed and within the age range of $20-55$ years. The Edinburgh Handedness Inventory was used to assess handedness (51). Patients were included in the study if they met the following inclusion criteria: (1) acute episode of MDD of unipolar subtype and 
a score of 18 or higher on the HRSD, and (2) free of psychotropic medication for a minimum of 3 weeks at recruitment. Exclusion criteria were: (1) axis I disorders such as bipolar disorder, anxiety disorder, or psychosis, (2) history of substance abuse within 6 months of study participation, (3) borderline personality disorder, (4) medical or neurological disorders, (5) severe suicidal symptoms, (6) failure to respond to three trials of antidepressant medication, or (7) contraindications for MRI (metal implants, pregnancy, etc.).

Nineteen HCs, matched by age and gender, were also recruited for the study through advertisements. These participants were screened using the Structured Clinical Interview for DSM-IV Axis I Disorders, non-patient version, to ensure they did not have previous or current Axis I psychiatric disorders (52) or any family history of Axis I disorders by self-report. The demographics of the MDD patients and HC are summarized in Table 1. Ethics approval was obtained from the local review board, and informed consent was obtained from all subjects prior to their participation in the study.

\section{MRI DATA ACQUISITION}

Images from 63 of the 74 participants were collected using a 3-T General Electric MR scanner (Signa VHi; General Electric Healthcare, Waukesha, WI, USA) equipped with an eight-channel, phased-array head coil. Two resting-state fMRI scans of $230 \mathrm{~s}$ (a total of $7 \mathrm{~min} 40 \mathrm{~s}$ ) in duration were acquired using a singleshot gradient-recalled echo, echo planar imaging sequence (115 volumes, TR/TE $=2000 / 30 \mathrm{~ms}, 24 \mathrm{~cm} \times 24 \mathrm{~cm}$ field of view, flip angle $=65^{\circ}, 30$ slices of $4 \mathrm{~mm}$ thickness). A T1-weighted structural $\mathrm{MRI}\left(\mathrm{TR}=9.2 \mathrm{~ms}, \mathrm{TE}=\right.$ Minimum, flip angle $=20^{\circ}, 180$ slices of thickness $1 \mathrm{~mm}$ ) was also acquired for anatomical registration of the fMRI data following analysis. Images of $11 \mathrm{MDD}$ patients were collected using $3 \mathrm{~T}$ General Electric MR scanner (Discovery MR 750) using the same parameters and protocol. Participants were required to remain still in the MRI scanner with their eyes open and fixated on a black crosshair at the center of a projection screen. The participants were instructed to relax, not think about anything in particular, and not to fall asleep.

\section{DATA ANALYSIS}

\section{Pre-processing of resting-state fMRI data}

All image data were analyzed using the FMRIB Software Library ${ }^{1}$ (FSL). Pre-processing of data included skull/scalp removal, slice timing correction, motion correction, spatial smoothing, and temporal high-pass filtering. MCFLIRT (53) was used to correct for head motion, using rigid-body transformations. Before any multisession or multi-subject analyses were performed, fMRI data were registered to the anatomical images as well as to the standard Montreal Neurological Institute (MNI) template. This was accomplished using FMRIB's Linear Image Registration Tool (FLIRT) $(53,54)$. Slice timing correction was done by using (Hanningwindowed) sinc interpolation to shift each time series by an appropriate fraction of the TR relative to the middle of the TR period. Spatial smoothing was done by applying a Gaussian filter

\footnotetext{
${ }^{1}$ http://www.fmrib.ox.ac.uk/fsl/
}

Table 1 | Demographic and clinical characteristics for the study sample.

\begin{tabular}{lll}
\hline & MDD $(\boldsymbol{n}=\mathbf{5 5})$ & $\begin{array}{l}\text { Healthy controls } \\
(\boldsymbol{n}=\mathbf{1 9})\end{array}$ \\
\hline Age (years) & $36.5 \pm 10.41$ & $32.89 \pm 9.97$ \\
Gender & $22 \mathrm{Male} / 33$ female & $8 \mathrm{Male} / 11$ female \\
Education (years) & $14.67 \pm 2.79$ & $14.89 \pm 2.00$ \\
HRSD* & $21.41 \pm 4.18$ & $3.38 \pm 1.89$ \\
HAM-A* & $25.56 \pm 5.15$ & $3.63 \pm 1.70$ \\
Age of onset & $24.68 \pm 10.41$ & - \\
Number of episodes & $2.67 \pm 3.86$ & - \\
Duration of current & $50.29 \pm 63.35$ & - \\
episode (months) & & \\
\hline
\end{tabular}

$M D D$, major depression disorder; HRSD, Hamilton rating scale for depression; $H A M-A$, Hamilton anxiety rating scale. Except for gender, all values are mean $\pm S D$ (range), ${ }^{*} p<0.001$.

with a full-width at half maximum of $6 \mathrm{~mm}$. Then the images were temporally filtered by 100 -s high-pass filter.

Signal fluctuations in the white matter (WM) and cerebrospinal fluid (CSF) predominantly reflect non-neural fluctuations such as scanner instabilities, physiological artifacts, and subject motion $(55,56)$. These signals are different from the gray matter signal and may introduce temporal coherence that can lead to an overestimation of functional connectivity strength. Hence, these effects were removed by extracting the mean time series of WM and CSF by averaging signal over all voxels within a WM or CSF mask for each time point. The masks of WM and CSF were obtained from each patient's functional image by selecting voxels within WM and CSF, respectively. The extracted mean WM and CSF time series were then used as temporal covariates during linear regression statistical analysis. A global signal regression was not performed, as this may lead to spurious anti-correlations (57-59).

The left and right amygdala were first located on the standard MNI brain template, using the structures tool available in the FSLView Toolbox. Using FLIRT, these regions were then registered to each participant's native fMRI image. Average time courses from each of the right and left ROIs were then extracted from the pre-processed resting-state fMRI. Prior to the analysis of images, the synchrony between the left and right amygdala for all participants was determined using the Pearson correlation coefficient, and was compared between MDD patients and HCs (after Fisher $Z$ transformation) by Student's $t$-test.

\section{fMRI statistical analysis}

Statistical analyses of the imaging data were carried out using fMRI expert analysis (FEAT), which uses a data modeling technique based on the general linear model. The general linear model method used for first-level data (i.e., time series data from a single session) was FMRIB's improved linear model (FILM). For the current study, a regression model was created to include the left amygdala time series as a predictor and eight nuisance covariates (time series predictors for WM, CSF, and the six motion parameters). The result is an estimate of how well the temporal response of the left amygdala correlates with the temporal signal of each 
voxel in the brain. This process was repeated again for the right amygdala.

Using FMRIB's Local Analysis of Mixed Effects (FLAME) in FEAT, we performed higher-level analysis (e.g., analysis across sessions or across subjects or between groups). Given the documented effect of age and sex on resting-state functional connectivity between regions (60-62), these were used as covariates of no-interest in all the group analyses. In addition, to control for scanner-related variations, we used the scanner as the covariate of no-interest in the model. Considering lateralized structural and functional differences $(46,63,64)$, statistical parameter maps were generated for the right and left amygdala separately, to determine the mean of each of the patient and control groups, as well as the significant difference between groups. From these analyses, $Z$-statistic images were generated with a statistical threshold of $Z=2.3(p=0.01)$, and a cluster size threshold of at least 77 contiguous voxels corresponding to a corrected $p$-value of 0.05 as determined by Monte Carlo simulations using AlphaSim ${ }^{2}$.

\section{Clinical correlate analysis}

To explore the relation between clinical variables and the abnormalities in RSFC of the amygdala in MDD, we computed the Pearson correlation coefficients between the mean $Z$ values of clusters that showed significant difference in RSFC contrast maps of MDD versus $\mathrm{HC}$ and each of three clinical variables (HRSD score, HAM-A score, and duration of illness since onset). Considering the exploratory nature of the analysis, we used an uncorrected statistical threshold of $p \leq 0.05$ for each test.

\section{RESULTS}

\section{CLINICAL AND SOCIO-DEMOGRAPHIC DATA}

Age, sex, and education were not significantly different between the MDD patient group and the comparison HC group (Table 1). As expected, mean HRSD and HAM-A scores were significantly higher for the MDD group compared with HCs. Furthermore, there were no differences in clinical and socio-demographic characteristics between MDD patients scanned in first scanner and second scanner (Table A1 in Appendix). Although most MDD patients had a HRSD total score of 18 or above at the time of enrollment, the score of 11 patients dropped below 18 on the day of imaging, which was performed 3-7 days after enrollment. All MDD patients, with the exception of three, were previously exposed to antidepressant medications. Four female patients were taking oral contraceptives during the study. Although none of the MDD patients met the criteria for a current anxiety disorder, most of the patients expressed anxiety symptoms (mean HAM-A score $25.56 \pm 5.15)$.

\section{DIFFERENCES IN RSFC OF AMYGDALA BETWEEN MDD AND HC}

The analysis regarding the synchrony between the left and right amygdala showed that the left amygdala was highly synchronous with the right amygdala for both subject groups (MDD: $r=0.87 \pm 0.06$; controls: $r=0.85 \pm 0.11$ ), and did not differ significantly $(t=0.43, p=0.67)$. The RSFC analysis demonstrated

\footnotetext{
${ }^{2}$ http://afni.nimh.nih.gov/afni/doc/manual/AlphaSim
}

Table 2 | Brain regions exhibiting a significant difference between patients with MDD and $\mathrm{HC}$ in the resting-state functional connectivity with the left amygdala ( $p<0.05$, corrected for multiple comparisons).

\begin{tabular}{cccc}
\hline Region (hemisphere) BA Cluster size $\frac{\text { MNI coordinate Z-score }}{\frac{x y y}{y}}$ &
\end{tabular}

\begin{tabular}{lcrrrrl}
\hline HC $>$ MDD & & & & & & \\
VLPFC (L) & $44 / 45$ & 99 & -54 & 18 & -2 & 4.16 \\
Primary motor (L) & 4 & 90 & -26 & -30 & 62 & 2.82 \\
Caudate (L) & & 131 & -12 & 0 & 16 & 3.57 \\
Insula (R) & 13 & 100 & 40 & 10 & -6 & 2.98 \\
Precuneus (R) & 7 & 84 & 16 & -44 & 62 & 3.3 \\
Middle temporal (L) & 21 & 185 & -56 & -36 & -4 & 3.33 \\
Middle temporal (R) & 21 & 171 & 60 & -8 & -10 & 3.77 \\
Superior temporal (R) & 22 & 293 & 58 & 8 & -8 & 3.17 \\
Occipital cortex (R) & $17 / 18$ & 177 & 2 & -86 & -6 & 3.06 \\
Occipital cortex (L) & $17 / 18$ & 131 & -14 & -84 & -20 & 3.57 \\
Cerebellum (R) & & 333 & 46 & -74 & -32 & 3.35 \\
Cerebellum (L) & & 154 & -10 & -38 & -16 & 3.99 \\
MDD > HC & & & & & & \\
Premotor (R) & 6 & 122 & 38 & 8 & 66 & 4.01 \\
Temporal pole (R) & 38 & 95 & 34 & 12 & -24 & 3.29 \\
\hline
\end{tabular}

$B A$, Brodmann's area; $H C$, healthy controls; MDD, major depression disorder; MNI, Montreal Neurological Institute.

significantly decreased functional connectivity of both the right and left amygdala with regions including the caudate, insula, occipital regions, and cerebellum (Tables 2 and 3; Figures 1 and 2). The left amygdala exhibited decreased connectivity with additional regions including the vlPFC, precuneus, superior and middle temporal regions, and primary motor cortex in MDD patients compared to HC, suggesting a lateralized pattern of reduced RSFC in MDD. Besides reduced RSFC, both the left and right amygdala exhibited increased connectivity with the contralateral temporal poles in MDD patients, compared to HC. In addition, the left amygdala showed increased connectivity with premotor cortex, while the right amygdala showed increased connectivity with superior temporal region overlapping with inferior frontal cortex, suggesting a differential pattern of connectivity between the right and left amygdala.

\section{CORRELATION WITH CLINICAL VARIABLES}

Given that the left amygdala demonstrated reduced connectivity with more regions than the right amygdala, we performed a correlation analysis between the clinical variables and brain regions with maximum cluster size that showed reduced RSFC with left amygdala. Among the regions that showed increased amygdalar RSFC in MDD, the temporal poles were selected because of bilateral involvement. We found no correlation between clinical variables (HRSD, HAM-A, and illness duration) and left amygdala connectivity with left caudate, right insula, left vlPFC, right precuneus, right cerebellum, and right occipital region. However, we found a significant inverse correlation between the left amygdala-right temporal pole RSFC and the HRSD scores $(r=-0.31, p=0.02)$, and HAM-A scores $(r=-0.46, p<0.001)$, in MDD patients. 
Table 3 | Brain regions exhibiting a significant difference between patients with MDD and $\mathrm{HC}$ in the resting-state functional connectivity of the right amygdala ( $p<0.05$, corrected for multiple comparisons).

\begin{tabular}{|c|c|c|c|c|c|c|}
\hline \multirow[t]{2}{*}{ Region (hemisphere) } & \multirow[t]{2}{*}{ BA } & \multirow[t]{2}{*}{ Cluster size } & \multicolumn{3}{|c|}{ MNI coordinate } & \multirow[t]{2}{*}{ Z-score } \\
\hline & & & $\boldsymbol{x}$ & $y$ & $z$ & \\
\hline \multicolumn{7}{|l|}{$H C>M D D$} \\
\hline Insula (R) & 13 & 128 & 34 & 2 & 8 & 3.13 \\
\hline Caudate (L) & & 106 & -12 & 0 & 16 & 3.05 \\
\hline Cuneus (R) & 17 & 218 & 24 & -82 & 10 & 3.14 \\
\hline Occipital/cuneus (L) & 17 & 122 & -16 & -94 & 24 & 3.37 \\
\hline Cerebellum (R) & & 153 & 48 & -64 & -32 & 3.31 \\
\hline \multicolumn{7}{|l|}{ MDD $>$ HC } \\
\hline Temporal pole (L) & 38 & 81 & -34 & 10 & -24 & 2.84 \\
\hline Superior temporal, & 21 & 93 & -58 & -2 & -34 & 2.96 \\
\hline
\end{tabular}

inferior frontal $(\mathrm{L})$

BA, Brodmann's area; HC, healthy controls; MDD, major depression disorder; MNI, Montreal Neurological Institute.

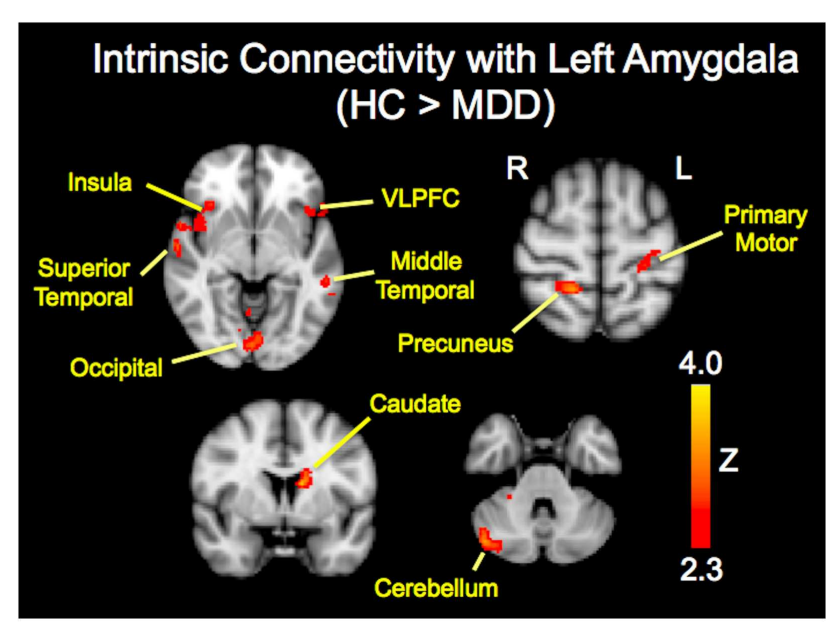

FIGURE 1 | Brain regions showing reduced resting-state functional connectivity with the left amygdala in depressed patients compared with healthy controls. Colored areas indicate significant $Z$-scores as determined by fMRI. Statistical threshold of $Z=2.3(p=0.01)$.

Furthermore, the HRSD and HAM-A scores were found to be significantly correlated ( $r=0.31, p=0.02$ ) probably due to overlapping of anxiety items in both scales and psychopathology of anxiety and depressive symptoms in MDD. This might partly explain the correlation of both test scores with RSFC between left amygdala and right temporal pole. No significant correlation was found between current illness duration and left amygdala intrinsic connectivity with right temporal pole $(r=-0.05, p=0.74)$.

\section{DISCUSSION}

In this study, we used seed-based (hypothesis driven) methodology to examine abnormalities in intrinsic connectivity of amygdala in patients with MDD. Confirming our hypothesis, our current

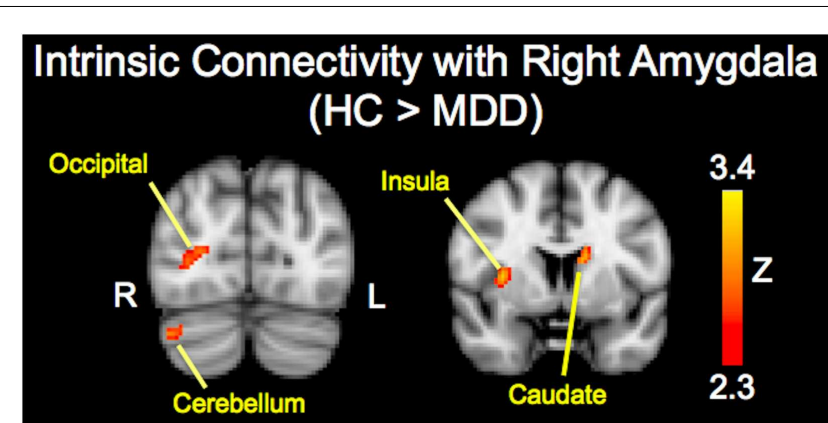

FIGURE 2 | Brain regions showing reduced resting-state functional connectivity with the right amygdala in depressed patients compared with healthy controls. Colored areas indicate significant $Z$-scores as determined by fMRI. Statistical threshold of $Z=2.3(p=0.01)$

data demonstrated reduced intrinsic connectivity of the amygdala with several brain regions implicated in depression including vlPFC, caudate, insula, precuneus, superior temporal gyrus (STG), occipital cortex, and cerebellum in MDD patients compared to HCs. However, we did not find abnormalities in intrinsic connectivity of the amygdala with medial prefrontal regions or ACC. Interestingly, MDD patients showed greater intrinsic connectivity between bilateral amygdala and contralateral temporal poles than healthy subjects and furthermore, intrinsic connectivity of the amygdala with right temporal pole was negatively correlated with depression severity and anxiety scores. Our results provide the first empirical evidence for wide-spread reduction in intrinsic connectivity of the amygdala with several functional networks involved in bottom-up emotional processing and regulation such as the ventral neural system (vlPFC, insula, and caudate), default mode network (precuneus), social cognition (STG), and visual and cerebellar regions.

\section{REDUCED INTRINSIC CONNECTIVITY WITH VENTRAL NEURAL SYSTEM}

It is worth noting that we observed decreased intrinsic connectivity between the amygdala and the right insula using seed-based analysis, which is consistent with previous findings using an ICA approach (36). Similarly, a decrease in intrinsic connectivity (negative connectivity) was observed between the amygdala seed region and fronto-insular region in MDD subjects corroborating our findings of aberrant intrinsic connectivity between amygdala and insula in depression (42). The amygdala and insula have common roles in processing saliency, emotion, and attention and have extensive reciprocal anatomical connections $(13,65,66)$. Patients with MDD exhibit hypoactive insula during the resting-state, as well as during emotional task conditions such as negative mood congruent processing, affective switching and in emotional judgment, suggesting biased saliency processing of emotional stimuli in $\operatorname{MDD}(67,68)$. Given the hyperactive amygdala and hypoactive insula in MDD, our findings of reduced amygdala-insula RSFC may suggest disruption in bottom-up saliency processing of negative emotion resulting in reduced self awareness of negative affect feelings, thus leading to negative bias in MDD. Additionally, the reduced amygdala-insula connectivity may hamper the formation of signals in insula to cortical input through the insula-dACC 
circuit affecting the top-down inhibition of the amygdala. In support of this view, previous work has shown that anterior insula might causally influence activity of dACC, the default mode network and central executive networks (69-71).

The observed attenuated connectivity between the amygdala and caudate highlights the disruptions of limbic-striatalthalamo-cortical circuits in MDD. Our results are in line with evidence from several neuroimaging studies suggesting the critical involvement of limbic-striatal-thalamo-cortical circuits in the pathophysiology of $\operatorname{MDD}(72,73)$. Both the amygdala and striatum have been implicated in motivational behavior and processing of both positive and negative emotions $(13,74)$. The amygdala has wide-spread projections to several components of the striatum including the nucleus accumbens and caudate nucleus (75). Thus, the amygdalostriatal anatomical and functional connectivity may be crucial in the formation of signals for cortical input to the premotor, medial, and lateral PFC for motivation and behavioral responses appropriate for the emotional state. Our results showing reduced connectivity between the bilateral amygdala and the left caudate, but not the right caudate, reflects decreased behavioral responses specific to processing negative emotions, as the left striatum is predominantly involved in processing negative emotions and right striatum in processing of positive emotions (68).

Within the prefrontal regions, we found decreased connectivity between amygdala and vlPFC, which has been implicated in processing emotional salience, motivation, and in the regulation of emotional and behavioral responses by modulating emotionrelated brain regions such as the amygdala and ventral striatum (76-80). Our results showing reduced connectivity with Broca's area (left vlPFC BA 44) may reflect impaired interactions between linguistic and cognitive systems (BA 47, 46) that are crucial for emotional evaluation and regulation (81). vlPFC BA 45b is closely related to the orbital frontal network, which functions as part of the ventral neural system for the bottom-up emotional processing and modulation, whereas vlPFC BA 45a has connections and interactions with the dorsal neural system (dlPFC BA 46, 9, 10), which is crucial for top-down emotional regulation (7). Our results showing reduced amygdala connectivity with the vlPFC (BA 44/45) may suggest impaired bottom-up as well as top-down regulation in MDD. This is in line with previous studies reporting decreased activity of vlPFC during voluntary emotional regulation and also decreased effective connectivity between the amygdala and vlPFC in $\operatorname{MDD}(29,82)$.

Contrary to previous studies, we did not find abnormalities in intrinsic connectivity between the amygdala and the medial PFC or ACC $(36,41)$. The discrepancies between these studies and our study could be ascribed to differences in patient samples and analysis methods. Our patient sample was exposed to medication, and we used a seed-based method, whereas the study by Veer et al. (36) involved depressed patients not exposed to medication and used data-driven ICA methods. Lui et al. (41), using a seed-based approach, showed decreased RSFC between the amygdala and the ACC in non-refractory MDD patients compared to the refractory depression (non-response to two adequate trials) or HCs. Non-refractory MDD patients were drug naïve prior to the study and improved with one or two trials of antidepressant treatment, whereas most of our patients (except three) were exposed to antidepressants and failed to improve with one or two antidepressants trials prior to their participation in this study. Another important source of discrepancy might be related to heterogeneity in amygdala connectivity in MDD (24).

\section{HYPOCONNECTIVITY WITH POSTERIOR REGIONS}

The precuneus is a key part of the default mode network, a neural substrate for visual imagery and episodic memory recall, and plays a major role in self-referential processing $(34,83)$. Taking into account that spontaneous activity in the amygdala negatively predicted activity in regions that are involved in cognitive control of emotions such as lateral PFC, posterior cingulate cortex, and precuneus in healthy subjects (46), and our results in conjunction with previous findings of decreased connectivity within the left amygdala-precuneus region in MDD patients (41) may be indicative of disruptions in bottom-up modulation of emotional processing.

The cerebellum, although underemphasized in the neural model of depression, has been implicated in mood disorders and could play a key role in emotional perception and cognitive processing (84). Electrical stimulation of the cerebellum in animals evoked responses in several brain regions involved in emotional and cognitive processing including PFC, ACC, amygdala, and hippocampus (85). Although it remains unclear whether the cerebellum has a direct inhibitory effect on amygdala responses to negative emotion or whether the amygdala has a modulatory effect on cerebellum, considering the extensive anatomical and functional connections of the cerebellum with prefrontal and limbic regions and the intrinsic connections between centromedial amygdala and cerebellum, our results suggest that amygdala-cerebellar connectivity may play a role in pathophysiology of depression (84).

The amygdala has extensive reciprocal anatomical connections with the visual cortex (86). Emotionally loaded visual stimuli have been shown to increase activation in the amygdala and visual cortex (87). Several imaging studies have demonstrated a possible modulatory role of the amygdala upon visual cortex in processing emotional faces $(88,89)$ probably through amygdalar efferents to the visual association cortex. Abnormalities in visual cortical regions have also been reported in mood disorders $(21,90)$. Visual cortex responses to sad faces predicted treatment outcome of antidepressant treatment in MDD $(90,91)$. Taken together, our results showing attenuated RSFC of amygdala with visual cortical regions (BA 17, 18) suggest disruption in bottom-up processing of emotionally laden visual stimuli in MDD.

\section{ABNORMAL CONNECTIVITY WITH TEMPORAL REGIONS}

The STG, temporal pole, and amygdala have been implicated in emotional processing and social cognition such as Theory of Mind (92, 93). Theory of Mind refers to our ability to understand and predict other people's social behavior by attributing to them independent mental states such as beliefs and desires (94). The STG has been reported to be active during explicit social judgment task whereas the amygdala seems to be involved in rapid automatic implicit judgment of socially salient stimuli (92). Furthermore, MDD patients have also shown decreased STG volume (95), neural activity at rest or in response to sad stimuli (67) suggesting the involvement of STG in the pathophysiology of 
MDD. Hence our results showing reduced connectivity between the amygdala and the STG may reflect impaired social and emotional processing in MDD. Interestingly, the decreased intrinsic connectivity was observed only with contralateral STG although its functional significance remains unclear. The observed increased intrinsic connectivity with temporal pole may represent compensatory mechanisms, as we found significant negative correlations between RSFC with temporal pole and severity of depression and anxiety scores.

\section{RIGHT AND LEFT AMYGDALA CONNECTIVITY}

Our results showed lateralized patterns of reduced connectivity for the left amygdala in MDD. The left amygdala demonstrated reduced connectivity with more cortical regions (vlPFC, precuneus, and temporal regions) than the right amygdala in MDD. These lateralized abnormalities in amygdala connectivity may reflect the structural and functional differences reported in the literature. The centromedial division of the left amygdala is 25\% smaller than the right amygdala (96), and meta-analytic studies have indicated more activation in left than right amygdala during emotional processing $(64,97)$ probably due to reduced habituation rate and elaborate cognitive and language processing (98, 99). Additionally, there is evidence that top-down responses may modulate only the left amygdala, whereas bottom-up responses may involve both amygdala (12). Consistent with this framework, our results showed reduced connectivity of the left amygdala with cortical regions linked to cognitive and language functioning and top-down regulation. However, the literature regarding the laterality of amygdala dysfunction in MDD is mixed, with results showing on the left, the right, and bilaterally $(41,100,101)$. Additional studies are needed to further investigate the question of laterality in intrinsic connectivity of the amygdala in MDD.

\section{LIMITATIONS}

There are several limitations that should be considered when interpreting these results. First, unbalanced design may have violated the assumptions underlying group analysis especially homogeneity of variance resulting in statistical error. Hence the results need to be replicated in a balanced design. Second, interscanner and intersubject variability would have affected our results as $11 \mathrm{MDD}$ subjects were scanned in a different scanner. However, we covaried for scanner differences and characteristics of MDD subjects scanned in two scanners were comparable. Third, a seed-based method limits our ability to examine functional connectivity patterns on a whole brain scale beyond the selected regions of interest. Fourth, temporal correlation analysis limits our inference on the directionality or causality of the abnormalities within the regions that are functionally connected. In order to accurately determine the directionality of altered resting-state functional connectivity of the amygdala, we must perform an effective connectivity study using methods such as Granger causality analysis (102-104). Furthermore, the observed reduced intrinsic connectivity of the amygdala could be a maladaptive response to a hyperactive amygdala. Future studies should take an integrative approach to examine the relation between resting-state activity and connectivity and how these may relate to the psychopathology of depression and progression of illness or clinical staging from vulnerability to refractory depression. Fifth, although patients were unmedicated at the time of imaging, prior exposure to long-term antidepressant medication might have an enduring effect on RSFC. Our results therefore need to be confirmed in drug naïve MDD patients. Sixth, since the participants in this study had moderate depression, our findings may not generalize to patients with severe depression. Seventh, the amygdala has three subdivisions (i.e., laterobasal, centromedial, and superficial) with distinct functional connections with limbic and cortical regions (46); we did not examine each division separately. However, our results indicate abnormalities in intrinsic connectivity of all three subdivisions of the amygdala in MDD patients given that in healthy subjects, resting-state activity in the laterobasal, centromedial, superficial subdivisions predicted activity in temporal/frontal regions, striatum/cerebellum, and limbic structures, respectively (46). Eighth, all the observed abnormalities may not reflect psychopathology of depression (state marker) as some may be related to vulnerability for depression (trait marker). Longitudinal prospective studies with treatment intervention are needed to determine state and trait related abnormalities in the intrinsic connectivity of the amygdala in MDD. Finally, it is possible that the group differences in worrying and anxiety at the time of scanning may have influenced our results as the anxiety state may increase resting-state activity in amygdala. However, it remains unclear whether increase in resting-state activity of amygdala may result in decrease in intrinsic connectivity or both are independent process. Future studies should address this question by investigating resting-state amygdala activity and FC in the same cohort.

\section{CONCLUSION}

The present study extended the findings of previous studies by investigating altered RSFC of the amygdala with the entire brain using a seed-based approach in MDD patients compared to HCs. Our study suggests abnormally decreased intrinsic connectivity between the amygdala and regions of the ventral neural system (vlPFC, insula, and caudate), which are known to be involved in bottom-up modulation of emotional processing and regions involved in self and social cognition (precuneus and STG) as well as posterior regions (occipital and cerebellum) implicated in emotional and cognitive processing. This abnormally decreased intrinsic connectivity of the amygdala could provide a neural network basis for a dysregulated, hyperactive amygdala, and for depressive symptoms including negative bias, impaired social cognition, and diminished motivational and behavioral responses in major depression.

\section{ACKNOWLEDGMENTS}

This work was supported by investigator initiated grant by Astra Zeneca to Dr. Rajamannar Ramasubbu. The infrastructure for this study was funded through the Hotchkiss Brain Institute for Neuroimaging Research Unit at Seaman Family MRI Research Centre-Foothills Hospital, Calgary. The authors wish to thank all participants who volunteered their time to participate in this study.

\section{REFERENCES}

\footnotetext{
1. Conwell Y, Duberstein PR, Cox C, Herrmann JH, Forbes NT, Caine ED. Relationships of age and axis I diagnoses in victims of completed suicide: a psychological autopsy study. Am J Psychiatry (1996) 153:1001-8.
} 
2. Cuijpers P, Smit F. Excess mortality in depression: a meta-analysis of community studies. J Affect Disord (2002) 72:227-36. doi:10.1016/S0165-0327(01) 00413-X

3. Kessler RC, Birnbaum HG, Shahly V, Bromet E, Hwang I, McLaughlin KA, et al. Age differences in the prevalence and co-morbidity of DSM-IV major depressive episodes: results from the WHO World Mental Health Survey Initiative. Depress Anxiety (2010) 27:351-64. doi:10.1002/da.20634

4. Collins PY, Patel V, Joestl SS, March D, Insel TR, Daar AS, et al. Grand challenges in global mental health. Nature (2011) 475:27-30. doi:10.1038/475027a

5. Nemeroff CB. Prevalence and management of treatment-resistant depression. J Clin Psychiatry (2007) 68(Suppl 8):17-25.

6. Mayberg HS. Modulating dysfunctional limbic-cortical circuits in depression: towards development of brain-based algorithms for diagnosis and optimised treatment. Br Med Bull (2003) 65:193-207. doi:10.1093/bmb/65.1.193

7. Price JL, Drevets WC. Neurocircuitry of mood disorders. Neuropsychopharmacology (2010) 35:192-216. doi:10.1038/npp.2009.104

8. Phillips ML, Drevets WC, Rauch SL, Lane R. Neurobiology of emotion perception I: the neural basis of normal emotion perception. Biol Psychiatry (2003) 54:504-14. doi:10.1016/S0006-3223(03)00168-9

9. Phillips ML, Ladouceur CD, Drevets WC. A neural model of voluntary and automatic emotion regulation: implications for understanding the pathophysiology and neurodevelopment of bipolar disorder. Mol Psychiatry (2008) 13(829):833-57. doi:10.1038/mp.2008.65

10. Ochsner KN, Silvers JA, Buhle JT. Functional imaging studies of emotion regulation: a synthetic review and evolving model of the cognitive control of emotion. Ann N Y Acad Sci (2012) 1251:E1-24. doi:10.1111/j.1749-6632.2012. 06751.x

11. Phillips ML, Drevets WC, Rauch SL, Lane R. Neurobiology of emotion perception II: implications for major psychiatric disorders. Biol Psychiatry (2003) 54:515-28. doi:10.1016/S0006-3223(03)00171-9

12. Ochsner KN, Ray RR, Hughes B, McRae K, Cooper JC, Weber J, et al. Bottom-up and top-down processes in emotion generation: common and distinct neural mechanisms. Psychol Sci (2009) 20:1322-31. doi:10.1111/j.1467-9280.2009. 02459.x

13. Lindquist KA, Wager TD, Kober H, Bliss-Moreau E, Barrett LF. The brain basis of emotion: a meta-analytic review. Behav Brain Sci (2012) 35:121-43. doi:10.1017/S0140525X11000446

14. LeDoux JE. Emotion circuits in the brain. Annu Rev Neurosci (2000) 23:155-84. doi:10.1146/annurev.neuro.23.1.155

15. Phelps EA, LeDoux JE. Contributions of the amygdala to emotion processing: from animal models to human behavior. Neuron (2005) 48:175-87. doi:10.1016/j.neuron.2005.09.025

16. Perez-Jaranay JM, Vives F. Electrophysiological study of the response of medial prefrontal cortex neurons to stimulation of the basolateral nucleus of the amygdala in the rat. Brain Res (1991) 564:97-101. doi:10.1016/0006-8993(91) 91357-7

17. Likhtik E, Pelletier JG, Paz R, Pare D. Prefrontal control of the amygdala. J Neurosci (2005) 25:7429-37. doi:10.1523/JNEUROSCI.2314-05.2005

18. Ghashghaei HT, Hilgetag CC, Barbas H. Sequence of information processing for emotions based on the anatomic dialogue between prefrontal cortex and amygdala. Neuroimage (2007) 34:905-23. doi:10.1016/j.neuroimage.2006. 09.046

19. Drevets WC, Videen TO, Price JL, Preskorn SH, Carmichael ST, Raichle ME. A functional anatomical study of unipolar depression. J Neurosci (1992) 12:3628-41.

20. Drevets WC, Price JL, Bardgett ME, Reich T, Todd RD, Raichle ME. Glucose metabolism in the amygdala in depression: relationship to diagnostic subtype and plasma cortisol levels. Pharmacol Biochem Behav (2002) 71:431-47. doi:10.1016/S0091-3057(01)00687-6

21. Surguladze S, Brammer MJ, Keedwell P, Giampietro V, Young AW, Travis MJ, et al. A differential pattern of neural response toward sad versus happy facial expressions in major depressive disorder. Biol Psychiatry (2005) 57:201-9. doi:10.1016/j.biopsych.2004.10.028

22. Victor TA, Furey ML, Fromm SJ, Ohman A, Drevets WC. Relationship between amygdala responses to masked faces and mood state and treatment in major depressive disorder. Arch Gen Psychiatry (2010) 67:1128-38. doi:10.1001/archgenpsychiatry.2010.144
23. Biswal B, Yetkin FZ, Haughton VM, Hyde JS. Functional connectivity in the motor cortex of resting human brain using echo-planar MRI. Magn Reson Med (1995) 34:537-41. doi:10.1002/mrm.1910340409

24. Pezawas L, Meyer-Lindenberg A, Drabant EM, Verchinski BA, Munoz KE, Kolachana BS, et al. 5-HTTLPR polymorphism impacts human cingulateamygdala interactions: a genetic susceptibility mechanism for depression. Nat Neurosci (2005) 8:828-34. doi:10.1038/nn1463

25. Chen CH, Suckling J, Ooi C, Fu CH, Williams SC, Walsh ND, et al. Functional coupling of the amygdala in depressed patients treated with antidepressant medication. Neuropsychopharmacology (2008) 33:1909-18. doi:10.1038/ sj.npp. 1301593

26. Matthews SC, Strigo IA, Simmons AN, Yang TT, Paulus MP. Decreased functional coupling of the amygdala and supragenual cingulate is related to increased depression in unmedicated individuals with current major depressive disorder. J Affect Disord (2008) 111:13-20. doi:10.1016/j.jad.2008.05.022

27. Dannlowski U, Ohrmann P, Konrad C, Domschke K, Bauer J, Kugel H, et al. Reduced amygdala-prefrontal coupling in major depression: association with MAOA genotype and illness severity. Int J Neuropsychopharmacol (2009) 12:11-22. doi:10.1017/S1461145708008973

28. Erk S, Mikschl A, Stier S, Ciaramidaro A, Gapp V, Weber B, et al. Acute and sustained effects of cognitive emotion regulation in major depression. J Neurosci (2010) 30:15726-34. doi:10.1523/JNEUROSCI.1856-10.2010

29. Carballedo A, Scheuerecker J, Meisenzahl E, Schoepf V, Bokde A, Moller HJ, et al. Functional connectivity of emotional processing in depression. J Affect Disord (2011) 134:272-9. doi:10.1016/j.jad.2011.06.021

30. Lu Q, Li H, Luo G, Wang Y, Tang H, Han L, et al. Impaired prefrontal-amygdala effective connectivity is responsible for the dysfunction of emotion process in major depressive disorder: a dynamic causal modeling study on MEG. Neurosci Lett (2012) 523:125-30. doi:10.1016/j.neulet.2012.06.058

31. Perlman G, Simmons AN, Wu J, Hahn KS, Tapert SF, Max JE, et al. Amygdala response and functional connectivity during emotion regulation: a study of 14 depressed adolescents. J Affect Disord (2012) 139:75-84. doi:10.1016/j.jad. 2012.01.044

32. de Kwaasteniet B, Ruhe E, Caan M, Rive M, Olabarriaga S, Groefsema M, et al. Relation between structural and functional connectivity in major depressive disorder. Biol Psychiatry (2013) 74:40-7. doi:10.1016/j.biopsych.2012.12.024

33. Wang L, Hermens DF, Hickie IB, Lagopoulos J. A systematic review of restingstate functional-MRI studies in major depression. J Affect Disord (2012) 142:6-12. doi:10.1016/j.jad.2012.04.013

34. Raichle ME, Macleod AM, Snyder AZ, Powers WJ, Gusnard DA, Shulman GL. A default mode of brain function. Proc Natl Acad Sci U S A (2001) 98:676-82. doi:10.1073/pnas.98.2.676

35. Greicius MD, Flores BH, Menon V, Glover GH, Solvason HB, Kenna H, et al. Resting-state functional connectivity in major depression: abnormally increased contributions from subgenual cingulate cortex and thalamus. Biol Psychiatry (2007) 62:429-37. doi:10.1016/j.biopsych.2006.09.020

36. Veer IM, Beckmann CF, Van Tol MJ, Ferrarini L, Milles J, Veltman DJ, et al. Whole brain resting-state analysis reveals decreased functional connectivity in major depression. Front Syst Neurosci (2010) 4:41. doi:10.3389/fnsys.2010. 00041

37. Bohr IJ, Kenny E, Blamire A, O’Brien JT, Thomas AJ, Richardson J, et al. Resting-state functional connectivity in late-life depression: higher global connectivity and more long distance connections. Front Psychiatry (2012) 3:116. doi:10.3389/fpsyt.2012.00116

38. Li B, Liu L, Friston KJ, Shen H, Wang L, Zeng LL, et al. A treatment-resistant default mode subnetwork in major depression. Biol Psychiatry (2013) 74:48-54. doi:10.1016/j.biopsych.2012.11.007

39. Zhang J, Wang J, Wu Q, Kuang W, Huang X, He Y, et al. Disrupted brain connectivity networks in drug-naive, first-episode major depressive disorder. Biol Psychiatry (2011) 70:334-42. doi:10.1016/j.biopsych.2011.05.018

40. Zhu X, Wang X, Xiao J, Liao J, Zhong M, Wang W, et al. Evidence of a dissociation pattern in resting-state default mode network connectivity in first-episode, treatment-naive major depression patients. Biol Psychiatry (2012) 71:611-7. doi:10.1016/j.biopsych.2011.10.035

41. Lui S, Wu Q, Qiu L, Yang X, Kuang W, Chan RC, et al. Resting-state functional connectivity in treatment-resistant depression. Am J Psychiatry (2011) 168:642-8. doi:10.1176/appi.ajp.2010.10101419 
42. Tahmasian M, Knight DC, Manoliu A, Schwerthoffer D, Scherr M, Meng C, et al. Aberrant intrinsic connectivity of hippocampus and amygdala overlap in the fronto-insular and dorsomedial-prefrontal cortex in major depressive disorder. Front Hum Neurosci (2013) 7:639. doi:10.3389/fnhum.2013.00639

43. Anand A, Li Y, Wang Y, Wu J, Gao S, Bukhari L, et al. Activity and connectivity of brain mood regulating circuit in depression: a functional magnetic resonance study. Biol Psychiatry (2005) 57:1079-88. doi:10.1016/j.biopsych.2005.02.021

44. Anand A, Li Y, Wang Y, Lowe MJ, Dzemidzic M. Resting state corticolimbic connectivity abnormalities in unmedicated bipolar disorder and unipolar depression. Psychiatry Res (2009) 171:189-98. doi:10.1016/j.pscychresns.2008.03.012

45. Alexopoulos GS, Hoptman MJ, Yuen G, Kanellopoulos D, Seirup JK, Lim KO, et al. Functional connectivity in apathy of late-life depression: a preliminary study. J Affect Disord (2013) 149:398-405. doi:10.1016/j.jad.2012.11.023

46. Roy AK, Shehzad Z, Margulies DS, Kelly AM, Uddin LQ, Gotimer K, et al. Functional connectivity of the human amygdala using resting state fMRI. Neuroimage (2009) 45:614-26. doi:10.1016/j.neuroimage.2008.11.030

47. Association AP, editor. Diagnostic and Statistical Manual. Washington: American Psychiatric Association Press (2000).

48. First MB, Gibbon M, Williams JBW, editors. Structured Clinical Interview for DSM-IV-TR Axis I Disorders, Research Version, Patient Edition. (SCID-I/P). New York: Biometric Research, New York State Psychiatric Institute (2002).

49. Hamilton M. The assessment of anxiety states by rating. Br J Med Psychol (1959) 32:50-5. doi:10.1111/j.2044-8341.1959.tb00467.x

50. Hamilton M. A rating scale for depression. J Neurol Neurosurg Psychiatry (1960) 23:56-62. doi:10.1136/jnnp.23.1.56

51. Oldfield RC. The assessment and analysis of handedness: the Edinburgh inventory. Neuropsychologia (1971) 9:97-113. doi:10.1016/0028-3932(71)90067-4

52. First MB, Gibbon M, Williams JBW, editors. Structured Clinical Interview for DSM-IV-TR Axis I Disorders, Research Version, Non-patient Edition. (SCID$I / N P)$. New York: Biometric Research, New York Psychiatric Institute (2002).

53. Jenkinson M, Bannister P, Brady M, Smith S. Improved optimization for the robust and accurate linear registration and motion correction of brain images. Neuroimage (2002) 17:825-41. doi:10.1006/nimg.2002.1132

54. Jenkinson M, Smith S. A global optimisation method for robust affine registration of brain images. Med Image Anal (2001) 5:143-56. doi:10.1016/S13618415(01)00036-6

55. Dagli MS, Ingeholm JE, Haxby JV. Localization of cardiac-induced signal change in fMRI. Neuroimage (1999) 9:407-15. doi:10.1006/nimg.1998.0424

56. Windischberger C, Langenberger H, Sycha T, Tschernko EM, FuchsjagerMayerl G, Schmetterer L, et al. On the origin of respiratory artifacts in BOLD-EPI of the human brain. Magn Reson Imaging (2002) 20:575-82. doi:10.1016/S0730-725X(02)00563-5

57. Fox MD, Zhang D, Snyder AZ, Raichle ME. The global signal and observed anticorrelated resting state brain networks. J Neurophysiol (2009) 101:3270-83. doi:10.1152/jn.90777.2008

58. Murphy K, Birn RM, Handwerker DA, Jones TB, Bandettini PA. The impact of global signal regression on resting state correlations: are anti-correlated networks introduced? Neuroimage (2009) 44:893-905. doi:10.1016/j.neuroimage. 2008.09.036

59. Anderson JS, Druzgal TJ, Lopez-Larson M, Jeong EK, Desai K, Yurgelun-Todd D. Network anticorrelations, global regression, and phase-shifted soft tissue correction. Hum Brain Mapp (2011) 32:919-34. doi:10.1002/hbm.21079

60. Biswal BB, Mennes M, Zuo XN, Gohel S, Kelly C, Smith SM, et al. Toward discovery science of human brain function. Proc Natl Acad Sci U S A (2010) 107:4734-9. doi:10.1073/pnas.0911855107

61. Tomasi D, Volkow ND. Aging and functional brain networks. Mol Psychiatry (2012) 17(471):549-58. doi:10.1038/mp.2012.27

62. Filippi M, Valsasina P, Misci P, Falini A, Comi G, Rocca MA. The organization of intrinsic brain activity differs between genders: a resting-state fMRI study in a large cohort of young healthy subjects. Hum Brain Mapp (2013) 34:1330-43. doi:10.1002/hbm.21514

63. Phillips ML, Medford N, Young AW, Williams L, Williams SC, Bullmore ET, et al. Time courses of left and right amygdalar responses to fearful facial expressions. Hum Brain Mapp (2001) 12:193-202. doi:10.1002/1097-0193(200104) 12:4<193::AID-HBM1015>3.0.CO;2-A

64. Baas D, Aleman A, Kahn RS. Lateralization of amygdala activation: a systematic review of functional neuroimaging studies. Brain Res Brain Res Rev (2004) 45:96-103. doi:10.1016/j.brainresrev.2004.02.004
65. Augustine JR. Circuitry and functional aspects of the insular lobe in primates including humans. Brain Res Brain Res Rev (1996) 22:229-44. doi:10.1016/ S0165-0173(96)00011-2

66. Craig AD. How do you feel - now? The anterior insula and human awareness. Nat Rev Neurosci (2009) 10:59-70. doi:10.1038/nrn2555

67. Fitzgerald PB, Laird AR, Maller J, Daskalakis ZJ. A meta-analytic study of changes in brain activation in depression. Hum Brain Mapp (2008) 29:683-95. doi:10.1002/hbm.20613

68. Diener C, Kuehner C, Brusniak W, Ubl B, Wessa M, Flor H. A meta-analysis of neurofunctional imaging studies of emotion and cognition in major depression. Neuroimage (2012) 61:677-85. doi:10.1016/j.neuroimage.2012.04.005

69. Sridharan D, Levitin DJ, Menon V. A critical role for the right fronto-insular cortex in switching between central-executive and default-mode networks. Proc Natl Acad Sci U S A (2008) 105:12569-74. doi:10.1073/pnas.0800005105

70. Menon V, Uddin LQ. Saliency, switching, attention and control: a network model of insula function. Brain Struct Funct (2010) 214:655-67. doi:10.1007/ s00429-010-0262-0

71. Ham T, Leff A, De Boissezon X, Joffe A, Sharp DJ. Cognitive control and the salience network: an investigation of error processing and effective connectivity. J Neurosci (2013) 33:7091-8. doi:10.1523/JNEUROSCI.4692-12.2013

72. Drevets WC. Functional neuroimaging studies of depression: the anatomy of melancholia. Annu Rev Med (1998) 49:341-61. doi:10.1146/annurev.med.49. 1.341

73. Yeh PH, Zhu H, Nicoletti MA, Hatch JP, Brambilla P, Soares JC. Structural equation modeling and principal component analysis of gray matter volumes in major depressive and bipolar disorders: differences in latent volumetric structure. Psychiatry Res (2010) 184:177-85. doi:10.1016/j.pscychresns.2010. 07.007

74. Liu X, Hairston J, Schrier M, Fan J. Common and distinct networks underlying reward valence and processing stages: a meta-analysis of functional neuroimaging studies. Neurosci Biobehav Rev (2011) 35:1219-36. doi:10.1016/j.neubiorev. 2010.12.012

75. Russchen FT, Bakst I, Amaral DG, Price JL. The amygdalostriatal projections in the monkey. An anterograde tracing study. Brain Res (1985) 329:241-57. doi:10.1016/0006-8993(85)90530-X

76. Tucker DM, Luu P, Pribram KH. Social and emotional self-regulation. Ann N Y Acad Sci (1995) 769:213-39. doi:10.1111/j.1749-6632.1995.tb38141.x

77. Cabeza R, Nyberg L. Imaging cognition II: an empirical review of 275 PET and fMRI studies. J Cogn Neurosci (2000) 12:1-47. doi:10.1162/ 08989290051137585

78. Hariri AR, Bookheimer SY, Mazziotta JC. Modulating emotional responses: effects of a neocortical network on the limbic system. Neuroreport (2000) 11:43-8. doi:10.1097/00001756-200001170-00009

79. Fuster JM. The prefrontal cortex - an update: time is of the essence. Neuron (2001) 30:319-33. doi:10.1016/S0896-6273(01)00285-9

80. Aron AR. The neural basis of inhibition in cognitive control. Neuroscientist (2007) 13:214-28. doi:10.1177/1073858407299288

81. Hariri AR, Mattay VS, Tessitore A, Fera F, Weinberger DR. Neocortical modulation of the amygdala response to fearful stimuli. Biol Psychiatry (2003) 53:494-501. doi:10.1016/S0006-3223(02)01786-9

82. Rive MM, Van Rooijen G, Veltman DJ, Phillips ML, Schene AH, Ruhe HG. Neural correlates of dysfunctional emotion regulation in major depressive disorder. A systematic review of neuroimaging studies. Neurosci Biobehav Rev (2013) 37(10 Pt 2):2529-53. doi:10.1016/j.neubiorev.2013.07.018

83. Cavanna AE, Trimble MR. The precuneus: a review of its functional anatomy and behavioural correlates. Brain (2006) 129:564-83. doi:10.1093/brain/ awl004

84. Schmahmann JD. Disorders of the cerebellum: ataxia, dysmetria of thought, and the cerebellar cognitive affective syndrome. J Neuropsychiatry Clin Neurosci (2004) 16:367-78. doi:10.1176/appi.neuropsych.16.3.367

85. Heath RG, Dempesy CW, Fontana CJ, Myers WA. Cerebellar stimulation: effects on septal region, hippocampus, and amygdala of cats and rats. Biol Psychiatry (1978) 13:501-29.

86. Amaral DG, Insausti R. Retrograde transport of D-[3H]-aspartate injected into the monkey amygdaloid complex. Exp Brain Res (1992) 88:375-88. doi:10.1007/BF02259113

87. Dolan RJ, Morris JS, editors. The Functional Anatomy of Innate and Acquired Fear: Perspective from Neuroscience. New York: Oxford University Press (2000). 
88. Morris JS, Friston KJ, Buchel C, Frith CD, Young AW, Calder AJ, et al. A neuromodulatory role for the human amygdala in processing emotional facial expressions. Brain (1998) 121(Pt 1):47-57. doi:10.1093/brain/121.1.47

89. Rotshtein P, Malach R, Hadar U, Graif M, Hendler T. Feeling or features: different sensitivity to emotion in high-order visual cortex and amygdala. Neuron (2001) 32:747-57. doi:10.1016/S0896-6273(01)00513-X

90. Furey ML, Drevets WC, Hoffman EM, Frankel E, Speer AM, Zarate CA Jr. Potential of pretreatment neural activity in the visual cortex during emotional processing to predict treatment response to scopolamine in major depressive disorder. JAMA Psychiatry (2013) 70:280-90. doi:10.1001/2013. jamapsychiatry.60

91. Keedwell PA, Drapier D, Surguladze S, Giampietro V, Brammer M, Phillips M. Subgenual cingulate and visual cortex responses to sad faces predict clinical outcome during antidepressant treatment for depression. J Affect Disord (2010) 120:120-5. doi:10.1016/j.jad.2009.04.031

92. Gallagher HL, Frith CD. Functional imaging of 'theory of mind'. Trends Cogn Sci (2003) 7:77-83. doi:10.1016/S1364-6613(02)00025-6

93. Olson IR, Plotzker A, Ezzyat Y. The Enigmatic temporal pole: a review of findings on social and emotional processing. Brain (2007) 130:1718-31. doi:10.1093/brain/awm052

94. Frith CD, Frith U. The neural basis of mentalizing. Neuron (2006) 50:531-4. doi:10.1016/j.neuron.2006.05.001

95. Takahashi T, Yucel M, Lorenzetti V, Walterfang M, Kawasaki Y, Whittle S, et al. An MRI study of the superior temporal subregions in patients with current and past major depression. Prog Neuropsychopharmacol Biol Psychiatry (2010) 34:98-103. doi:10.1016/j.pnpbp.2009.10.005

96. Ball T, Rahm B, Eickhoff SB, Schulze-Bonhage A, Speck O, Mutschler I. Response properties of human amygdala subregions: evidence based on functional MRI combined with probabilistic anatomical maps. PLoS One (2007) 2:e307. doi:10.1371/journal.pone.0000307

97. Wager TD, Phan KL, Liberzon I, Taylor SF. Valence, gender, and lateralization of functional brain anatomy in emotion: a meta-analysis of findings from neuroimaging. Neuroimage (2003) 19:513-31. doi:10.1016/S1053-8119(03) 00078-8

98. Markowitsch HJ. Differential contribution of right and left amygdala to affective information processing. Behav Neurol (1998) 11:233-44. doi:10.1155/ $1999 / 180434$

99. Sergerie K, Chochol C, Armony JL. The role of the amygdala in emotional processing: a quantitative meta-analysis of functional neuroimaging studies. Neurosci Biobehav Rev (2008) 32:811-30. doi:10.1016/j.neubiorev. 2007.12.002

100. Abercrombie HC, Schaefer SM, Larson CL, Oakes TR, Lindgren KA, Holden JE, et al. Metabolic rate in the right amygdala predicts negative affect in depressed patients. Neuroreport (1998) 9:3301-7. doi:10.1097/00001756-19981005000028

101. Drevets WC, Price JL, Furey ML. Brain structural and functional abnormalities in mood disorders: implications for neurocircuitry models of depression. Brain Struct Funct (2008) 213:93-118. doi:10.1007/s00429-008-0189-x

102. Goebel R, Roebroeck A, Kim DS, Formisano E. Investigating directed cortical interactions in time-resolved fMRI data using vector autoregressive modeling and Granger causality mapping. Magn Reson Imaging (2003) 21:1251-61. doi:10.1016/j.mri.2003.08.026

103. Roebroeck A, Formisano E, Goebel R. Mapping directed influence over the brain using Granger causality and fMRI. Neuroimage (2005) 25:230-42. doi:10.1016/j.neuroimage.2004.11.017

104. Hamilton JP, Chen G, Thomason ME, Schwartz ME, Gotlib IH. Investigating neural primacy in major depressive disorder: multivariate Granger causality analysis of resting-state fMRI time-series data. Mol Psychiatry (2011) 16:763-72. doi:10.1038/mp.2010.46

Conflict of Interest Statement: The authors declare that the research was conducted in the absence of any commercial or financial relationships that could be construed as a potential conflict of interest.

Received: 12 November 2013; accepted: 04 February 2014; published online: 19 February 2014.

Citation: Ramasubbu R, Konduru N, Cortese F, Bray S, Gaxiola-Valdez I and Goodyear B (2014) Reduced intrinsic connectivity of amygdala in adults with major depressive disorder. Front. Psychiatry 5:17. doi: 10.3389/fpsyt.2014.00017

This article was submitted to Neuropsychiatric Imaging and Stimulation, a section of the journal Frontiers in Psychiatry.

Copyright (c) 2014 Ramasubbu, Konduru, Cortese, Bray, Gaxiola-Valdez and Goodyear. This is an open-access article distributed under the terms of the Creative Commons Attribution License (CC BY). The use, distribution or reproduction in other forums is permitted, provided the original author(s) or licensor are credited and that the original publication in this journal is cited, in accordance with accepted academic practice. No use, distribution or reproduction is permitted which does not comply with these terms. 


\section{APPENDIX}

Table A1 | Characteristics of MDD subjects scanned in two scanners.

\begin{tabular}{|c|c|c|c|}
\hline & Scanner $1(n=44)$ & Scanner $2(n=11)$ & $p$-Value \\
\hline Age (years) & $36.84 \pm 10.34$ & $36.09 \pm 10.45$ & 0.85 \\
\hline Gender & 16 Male/28 female & 6 Male/5 female & 0.32 \\
\hline Education (years) & $14.67 \pm 2.79$ & $14.89 \pm 2.00$ & 0.15 \\
\hline HRSD & $21.50 \pm 3.76$ & $20.55 \pm 4.32$ & 0.41 \\
\hline HAM-A & $23.93 \pm 4.62$ & $21.82 \pm 5.23$ & 0.19 \\
\hline Age of onset & $24.73 \pm 9.85$ & $26.55 \pm 13.74$ & 0.62 \\
\hline Number of episodes & $2.98 \pm 4.22$ & $1.64 \pm 1.80$ & 0.32 \\
\hline Duration of current episode (months) & $50.00 \pm 64.26$ & $25.36 \pm 51.60$ & 0.16 \\
\hline
\end{tabular}

MDD, major depression disorder; HRSD, Hamilton rating scale for depression; HAM-A, Hamilton anxiety rating scale. 